\title{
El colgajo miomucoso de la arteria facial para tratamiento de defectos intraorales. Reporte de 2 Casos
}

Martínez $A^{1}$. El colgajo miomucoso de la arteria facial para tratamiento de defectos intraorales. Rev Estomatol Herediana. 2012 Oct-Dic;22(4):228-31.

\section{RESUMEN.}

El tratamiento de defectos intraorales puede resultar complicado y mórbido cuando se usan colgajos distantes. En determinados casos el uso del colgajo miomucoso de la arteria facial (CMMAF) puede facilitar el cierre de defectos intraorales de tamaño pequeño y mediano. Presentamos 2 casos manejados con CMMAF para cierre de fistulas oronasal y vestíbulo antero inferior mandibular. La técnica es sencilla y no requiere entrenamiento extensivo ya que la anatomía de la zona es muy familiar para todo cirujano oral y maxilofacial. En conclusión el CMMAF debería considerarse como una de la primeras opciones para reconstruir defectos intraorales de cierto tamaño.

\section{Alan Yuri Martínez ${ }^{1}$}

Cirujano Oral y Maxilofacial. Profesor Clínico Asistente. MetroHealth Medical Center. Cleveland, Ohio.

\section{Correspondencia:}

Alan Yuri Martínez, D.D.S.

Cleveland, Ohio.

Correspondencia: aymartinez@gmail.com
Palabras clave: COLGAJO, FACIAL, RECONSTRUCCIÓN, INTRAORAL.

The facial artery myomucosal flap (FAMM) for the management of intraoral defects. Report of 2 cases. ABSTRACT

The management of intraoral defects can be complicated and carry significant morbidity when using distant flaps. In selected cases the use of a facial artery myomucosal flap (FAMM) facilitates the closure of small and medium size defects. We are reporting 2 cases in which a FAMM flap was used successfully to close an oronasal and orocutaneous fistulaes. The surgical technique is simple and does not require specialized training due to the familiarity with the anatomic region that all oral and maxillofacial surgeons have. In conclusion we believe the FAMM flap should be considered as one of the first reconstruction options for certain size intraoral defects.

Key words: FLAP, FACIAL, RECONSTRUCTION, INTRAORAL.

\section{Introducción}

El colgajo miomucoso de la arteria facial (CMMAF) fue introducido por primera vez por Pribaz et al en 1992 (1). Sin embargo muchos autores han publicado sobre el uso de colgajos miomucosos que incluyen el musculo buccinador $(2,3)$. Estos colgajos representan una excelente opción para el cirujano oral y maxilofacial ya que la morbilidad es mínima y el tejido transferido es mucosa. CMMAF puede ser diseñado con el pedículo vascular superior (flujo retrogrado) o con el pedículo vascular inferior (flujo anterógrado) dependiendo de la localización del defecto. En cualquiera de las dos opciones el colgajo es muy confiable y fácil de disecar.

\section{Anatomía quirúrgica}

Identificar y marcar los vasos faciales mediante un ultrasonido Doppler portátil. El diseño del col- gajo será basado en el tamaño y localización del defecto. Identificar la desembocadura del conducto parotídeo. Idealmente la incisión superior se debería de ubicar unos 5-10 mm por debajo del conducto parotídeo, la incisión anterior unos 10 mm por detrás de la comisura bucal posteriormente e inferiormente se determina de acuerdo a la cantidad de tejido necesaria para cubrir el defecto sin tensión. La incisión se lleva acabo a través de mucosa, submucosa y musculo buccinador con electro bisturí con cuidado de no dañar el conducto parotídeo. Una vez que se identifica la arteria y la vena facial (debajo del buccinador) se ligan y la disección se realiza cuidadosamente tratando de no dañar los vasos y de mantenerlos adheridos al musculo. Este plano en general es fácil de identificar ya que el musculo buccinador esta separado por la fascia bucofa- ríngea. Una vez que el colgajo ha sido elevado se ubica mediante un túnel submucoso o pediculado sobre el defecto dependiendo de la presencia de dientes y localización del lugar a reconstruir. Durante la inserción y sutura del colgajo hay que tener mucho cuidado en no dañar o doblar los vasos faciales. Si el defecto esta localizado en la región palatina es importante pasar unas suturas antes de colocar el colgajo en la región con menos visibilidad generalmente posterior superior. Si el colgajo es pediculado un segundo tiempo quirúrgico debe ser planeado idealmente 3 semanas después para dividir el pedículo y remover exceso de tejido.

\section{Presentación de casos}

Caso 1

Paciente masculino de 48 anos de edad con antecedentes de diabetes, fumador (por 20 años), hi- 
pertensión arterial y historia de un quiste apical en la región incisiva maxilar (enucleado en el año 2010), que posteriormente resultó en una fistula oronasal de aproximadamente $1.5 \mathrm{~cm}$ x $1.5 \mathrm{~cm}$ (Fig 1).

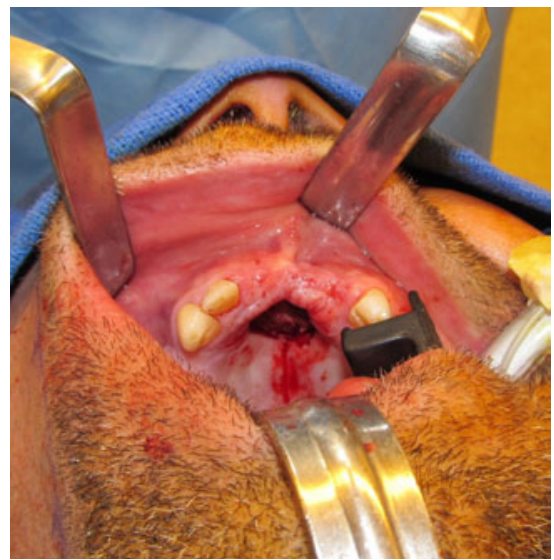

Fig 1. Fistula oronasal después de remover epitelio y realización de un colgajo marginal con cierre en si mismo.

El paciente usaba un obturador pero deseaba tratamiento definitivo mediante cirugía. En Agosto del 2012 el paciente fue llevado al quirófano y un CMMAF con pedículo vascular superior fue delineado (Fig 2).

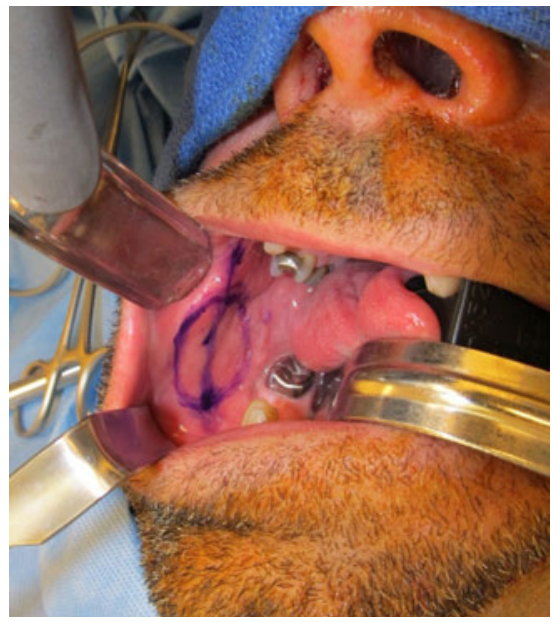

Fig 2. Curso de la arteria facial y demarcación de colgajo con plumón quirúrgico.

Lo primero a realizar fue una fistulectomia y realización de un colgajo marginal con cierre en si mismo. Posteriormente el curso de la arteria facial y CMMFA fue marcado con ayuda de un ultrasonido Doppler. Anestesia local con epinefrina fue inyectada superficialmente. La incisión fue llevada a cabo con electro bisturí hasta identificar la arteria facial en la parte inferior del colgajo desde aquí una disección cuidadosa fue realizada incluyendo los vasos faciales en el colgajo. Una vez que el colgajo fue elevado, se verifico el alcance sin tensión a la región palatina anterior (Fig 3).

En colgajo es fijado con suturas interrumpidas (Fig 4).

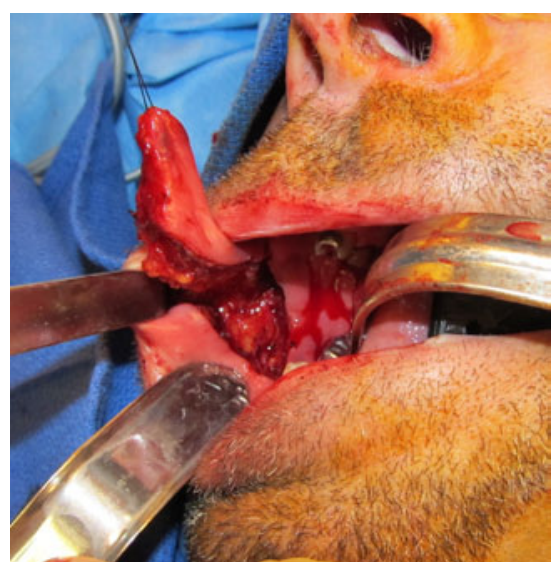

Fig 3. CMMAF elevado en su pedículo superior.

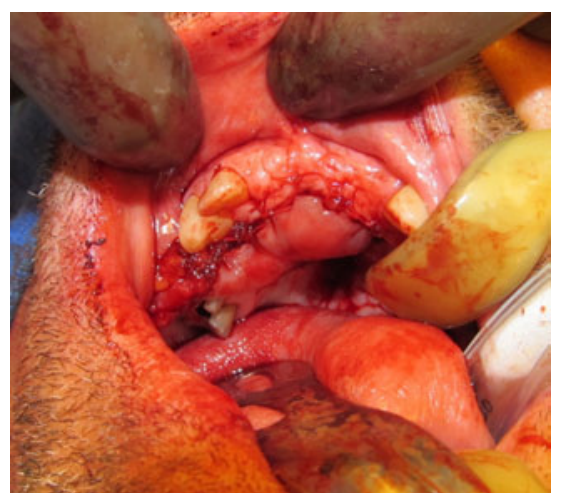

Fig 4. Colgajo suturado en posición. Nótese espacio edentulo por donde el pedículo fue transferido a zona receptora.

En el postoperatorio el paciente fue instruido en mantener una dieta blanda y excelente higiene oral. Tres semanas después el paciente se encontraba listo para liberación de pedículo y plastia de tejido excesivo (Figs 5 y 6 ).

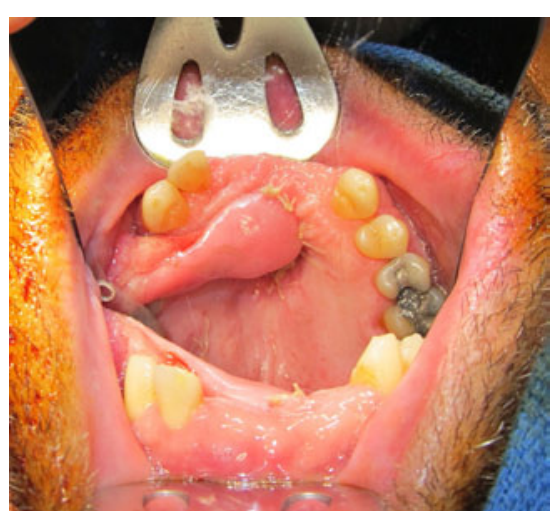

Fig 5. Semana 3 postoperatorio, el colgajo viable y listo para ser dividido.

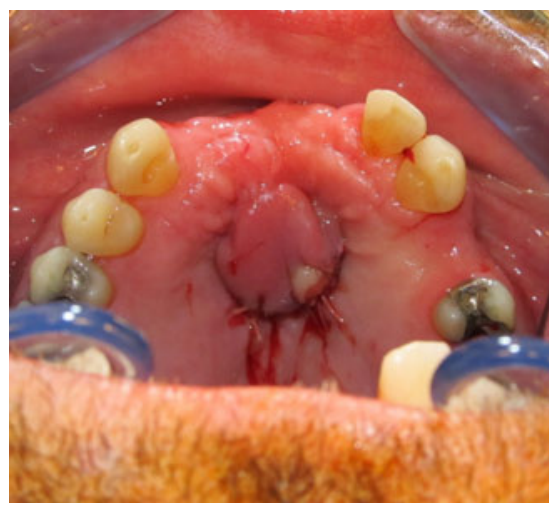

Fig 6. Colgajo después de su división y plastia.

Actualmente el paciente se encuentra asintomático y usando una prótesis parcial removible maxilar.

\section{Caso 2}

Paciente masculino de 55 anos con antecedentes de diabetes, hipertensión arterial y fractura mandibular debido a herida por arma de fuego (aproximadamente 20 años atrás). El recibió tratamiento con placas y tornillos de acero quirúrgico para reparar fracturas mandibulares. Recientemente sufrió aumento de volumen en región submentoniana derecha y submandibular izquierda con drenaje de material purulento y saliva en región submandibular (Fig 7).

El paciente fue preparado para retiro de placas y tornillos. Fistulectomias bilaterales y debido a la comunicación intraoral en el lado izquierdo un CMMFA fue diseñado 
con pedículo inferior (Fig 8).

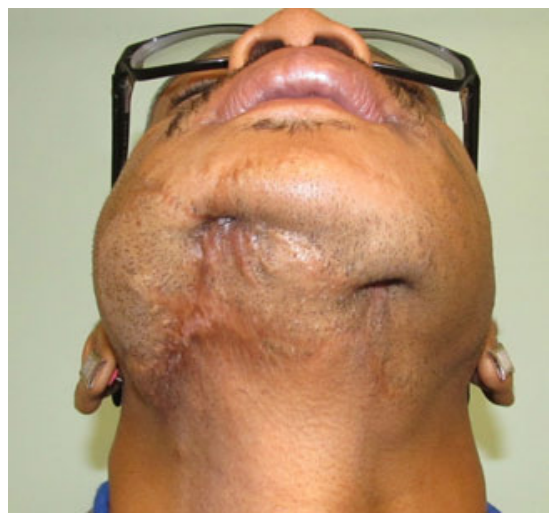

Fig 7. Fotografía pre quirúrgica. Nótese múltiples cicatrices y fistulas.

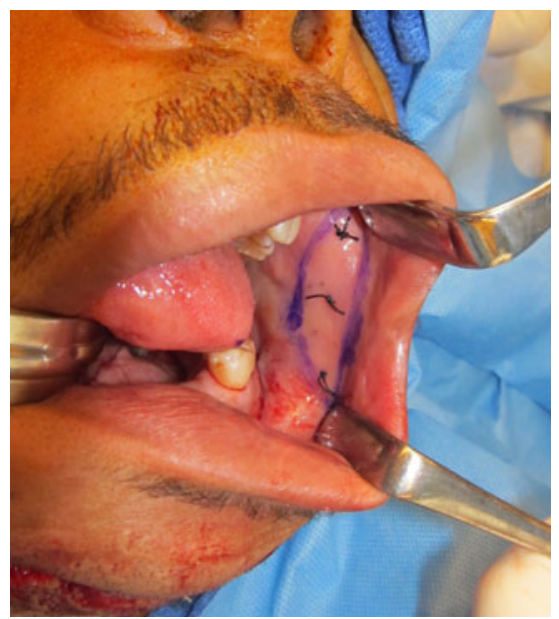

Fig 8. Diseño del colgajo. Los puntos de seda negra están marcando el tramo de la arteria facial.

Una vez que las placas y tornillos fueron retirados y las fistulectomias terminadas el CMMAF fue elevado pero comenzando por la región superior hasta identificar la arteria facial. Después el colgajo fue disecado con la misma técnica descrita anteriormente, pero con pedículo vascular inferior (Fig 9).

Debido a que la incisión para remover la placa mandibular se encontraba cerca de la base del colgajo. Se realizo una pequeña extensión para unir las dos incisiones y así evitar una segunda cirugía (Fig 10). El colgajo fue suturado en su lugar sin complicaciones.

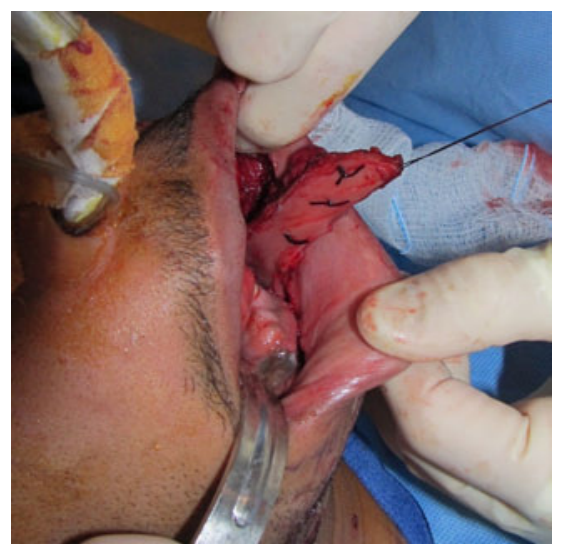

Fig 9. Colgajo elevado y movilizado hacia defecto del vestíbulo mandibular anterior.

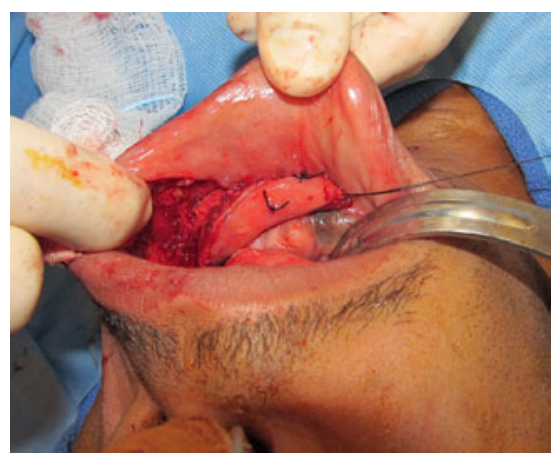

Fig 10. Colgajo en posición sin tensión.

El postoperatorio del paciente fue sin complicaciones y las fistulas se mantuvieron sin secreciones (Fig 11).

Intraoralmente el paciente presentó inicialmente limitación a la apertura bucal, pero con terapia física fue mejorando hasta llegar a una apertura normal (Fig 12).

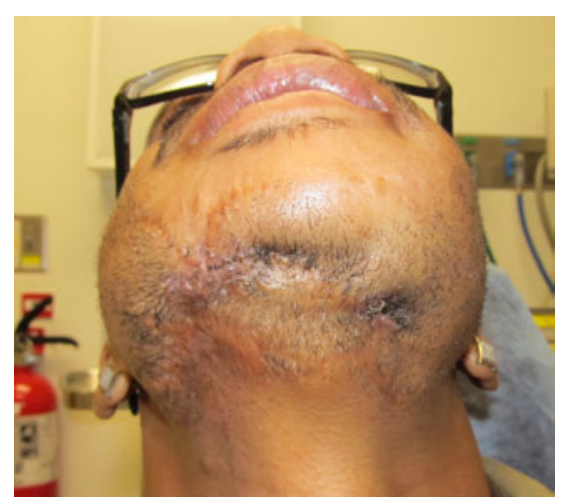

Fig 11. Postoperatorio 3 semanas.

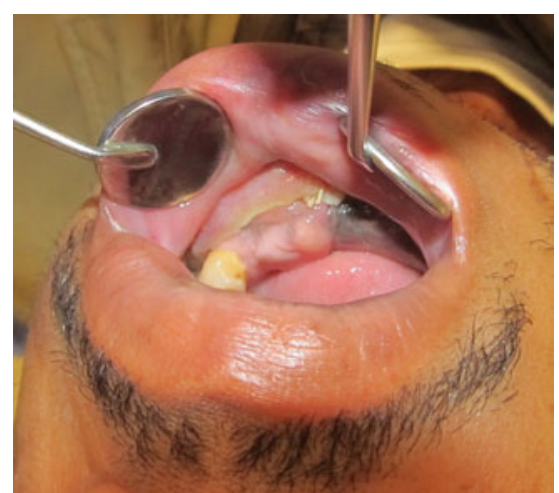

Fig 12. Colgajo viable, sin fistula extraoral (3 semanas postoperado).

\section{Discusión}

El cierre de defectos intraorales de cierto tamaño (hasta $3 \mathrm{~cm}$ de diámetro) puede realizarse mediante el uso de CMMAF, colgajos de mayor tamaño requerirían el uso de injertos de piel para cubrir zona dadora. Aumentando la morbilidad y por ende eliminando una de las ventajas que atribuimos a este colgajo. Para el manejo de defectos de mayor tamaño el uso de colgajos loco regionales como el pectoral mayor, supraclavicular, temporal, submentoniano o colgajos microvascularizados serian una mejor alternativa.

Un factor importante en pacientes oncológicos es el estado del cuello. La presencia de patología cervical maligna seria una contra indicación relativa debido a posible compromiso oncológico al momento de disecar la arteria facial para preservarla (4). El uso de CMMAF para reconstruir defectos intraorales posterior a resección de patología maligna en pacientes con cuello N0 ha sido publicado por diferentes autores $(4,5)$. La versatilidad de este colgajo para alcanzar y reconstruir defectos en el paladar (6, 7) y vestíbulo mandibular (8) han sido publicados recientemente por diferentes especialidades de todo el mundo. En nuestros casos ambos 
colgajos sobrevivieron sin complicaciones. El único problema que tuvimos fue una dehiscencia en el paciente \#1 en la región posterior superior del colgajo debido a un error técnico y no pasar todas las suturas en el paladar (sector posterior) previamente antes de asegurar el primer nudo. La dehiscencia fue cerrada sin complicaciones durante el segundo tiempo quirúrgico después de seccionar el pedículo.

\section{Conclusión}

El CMMAF es confiable, técnicamente simple de realizar y constituye una zona dadora mas para el cirujano oral y maxilofacial. Especialmente cuando los defectos a reconstruir son de tamaño pequeño a mediano.

\section{Referencias Bibliográficas}

1. Pribaz JJ, Stephens W, Crespo L, Gifford G. A new intraoralflap: facial artery musculomucosal (FAMM) flap. Plast Reconstr Surg 1992;90:421-9.

2. Bozola AR, Gasques JAL, Carriquiry CE, Cardoso de Oliveira M. The buccinator musculomucosal flap: anatomic study and clinical application. Plast Reconstr Surg 1989;84:250-7.

3. Zhao Z, Li S, Yan Y, Li Y, Yang $\mathrm{M}, \mathrm{Mu} \mathrm{L}$, et al. New buccinatormyomucosal island flap: anatomic study and clinical application. Plast Reconstr Surg 1999;104:55-64.

4. Bianchi B, Ferri A, Ferrari S, Copelli C, Sesenna E. Myomucosal cheek flaps : applications in intraoral reconstruction using three different techniques. Oral Surg
Oral Med Oral Pathol Oral Radiol Endod 2009;108:353-9.

5. Ferrari S, Balestreri A, Bianchi B, Multinu A, Ferri A, Sesenna E. Buccinator myomucosal island flap for reconstruction of the floor of the mouth. J Oral Maxillofac Surg 2008; 66:394-400.

6. Bonawitz SC, Duvvuri U. Robotic-Assisted FAMM flap for soft palate reconstruction. Laryngoscope. 2013 Apr;123(4):870-4.

7. Joshi A, Rajendraprasad JS, Shetty K. Reconstruction of intraoral defects using facial artery myomucosal flap. $\mathrm{Br} \mathrm{J}$ Plast Surg 2005;58:1061-6.

8. Hatoko M, Kuwahara M, Tamaka A, Yurugi S. Use of facial artery musculomucosal falp for closure of soft tissue defects of the mandibular vestibule. Int J Oral Maxillofac Surg 2002; 31:210-211. 